\title{
Lovastatin rescues human and mice cartilage disorders
}

\author{
Satoshi Kubota
}

Received: 30 January 2015 / Accepted: 30 January 2015 / Published online: 5 March 2015

(C) The International CCN Society 2015

\begin{abstract}
Statin, a well-known inhibitor of cholesterol synthesis, combats excessive FGF signaling and may rescue the affected. Relevance to CCN family function may be suspected therein.
\end{abstract}

Keywords FGF · FGFR · Statins · Achondroplasia · Thanatophoric dysplasia

Yamashita et al. (2014) describes an amazing effect of lovastatin, an inhibitor of cholesterol synthesis and protein prenylation, on genetic disorders caused by gain-of-function mutations of fibroblast growth factor receptor 3 (FGFR3). Employing a sophisticated technique for the generation of disease-specific induced pluripotent stem (iPS) cells, the authors successfully reproduced the cartilage of FGFR3 skeletal disorder patients. After the screening, lovastatin was found to efficiently rescue, not only the human cartilage form the patient in vitro, but also corresponding model mice in vivo. In the context of CCN family biology, this article is of particular interest, since lovastatin represses the production of CCN2 that is reported to enhance FGF signaling.

\section{References}

Yamashita A, Morioka M, Kishi H, Kimura T, Yahara Y, Okada M, Fujita K, Sawai H, Ikegawa S, Tsumaki N (2014) Statin treatment rescues FGFR3 skeletal dysplasia phenotypes. Nature 513:507-511
S. Kubota $(\bowtie)$

Okayama University Graduate School of Medicine, Dentistry and

Pharmaceutical Sciences, Okayama, Japan

e-mail: kubota1@md.okayama-u.ac.jp 